\title{
Kink effect and noise performance in isolated-gate InAs/AlSb High Electron Mobility Transistors
}

\author{
B. G. Vasallo ${ }^{1 *}$, H. Rodilla ${ }^{2}$, T. González ${ }^{1}$, G. Moschetti ${ }^{2}$ J. Grahn ${ }^{2}$, and J. Mateos ${ }^{1}$ \\ ${ }^{1}$ Dpto. Física Aplicada, Universidad de Salamanca, Plaza de la Merced s/n, 37008 \\ Salamanca, Spain \\ Phone: +34-923294436, Fax: +34-923294584*e-mail: bgvasallo@usal.es \\ ${ }^{2}$ Microwave Electronics Laboratory, Department of Microtechology and Nanoscience- \\ MC2, Chalmers University of Technology, SE412 Göteborg, Sweden \\ Departamento de Física Aplicada, Universidad de Salamanca,
}

\begin{abstract}
Kink effect can spoil the otherwise excellent low-noise performance of InAs/AlSb HEMTs. It has its origin in the pile up of holes (generated by impact ionization) taking place mainly at the drain side of the buffer, which leads to a reduction of the gateinduced channel depletion and results in a drain current enhancement. Our results indicate that the generation of holes by impact ionization and their further recombination lead to fluctuations in the charge of the hole pile up, which provoke an important increase of the drain-current noise, even when the kink effect is hardly perceptible in the output characteristics.
\end{abstract}




\section{Introduction}

Due to the high mobility of electrons in InAs and the excellent electron confinement in the channel, InAs/AlSb High Electron Mobility Transistors (HEMTs) are being developed for low-power, high-frequency and low-noise applications [1-7]. Nevertheless, there are some drawbacks to be eliminated, as the kink effect (an anomalous increase of the drain current $I_{D}$ when increasing the drain-to-source voltage $V_{D S}$ ), caused by impact ionization and the subsequent hole dynamics in the structure. Impact ionization events are extremely frequent because of the narrow band-gap of InAs, and the performance of these devices can be dramatically affected by kink effect. In particular, it leads to a reduction in the gain and a rise in the level of noise, limiting the utility of these devices for possible microwave or mm-wave applications. Strategies to reduce the negative consequences of kink effect on the operation of InAs/AlSb HEMTs are thus essential. For example, to overcome the appearance of the associated excessive gate leakage current related to impact-ionization generated holes, the conventional Schottky contact has been replaced by an isolated gate by means of a native oxide [7]. However, the reduction of the kink-effect related noise is still an issue. The purpose of this work is to analyze the influence of the kink effect on the noise performance of isolated-gate InAs/A1Sb HEMTs. With this aim, we make use of a semiclassical 2D ensemble Monte Carlo (MC) simulator [8] adequately adapted to correctly reproduce the experimental static, dynamic and noise behavior of InAs/AlSb devices in absence of kink effect (this is, for low values of $V_{D S}$ ) [9-11], in which the impact ionization and hole transport have been included [12-14]. The MC method is the most appropriate technique for the analysis of these devices, since electron transport can easily turn into ballistic or quasi-ballistic in the channel due to the huge mobility of InAs [9]. Moreover, with our MC method, a complete physical understanding of the kink phenomenon and its influence on the noise behavior in these devices can be achieved, thus providing helpful information for the development of Sb-HEMTs with improved immunity to this effect.

The paper is organized as follows. In Sec. II the physical model is detailed. The main results and their discussion are provided in Sec. III. Finally, in Sec. IV we draw the most important conclusions of this work. 


\section{Physical Model}

For the calculations we make use of an ensemble MC simulator self-consistently coupled with a 2D Poisson solver which incorporates the processes at the origin of the kink effect. The simulated structure is very similar to the experimental one described in Ref. [6], which has been used to calibrate our model by reproducing the static characteristics. The structure under analysis is a $225 \mathrm{~nm}$ T-gate recessed HEMT (figure 1), fabricated on a heterostructure consisting of a InP substrate (not simulated), a $800 \mathrm{~nm}$ AlSb buffer followed by a $15 \mathrm{~nm}$ thick InAs channel, two layers of AlSb (a $5 \mathrm{~nm}$ spacer and a $10 \mathrm{~nm}$ Schottky layer, separated by a $5 \times 10^{12} \mathrm{~cm}^{-2} \delta$-doped layer), and, finally, a $4 \mathrm{~nm}$ thick AlInAs and $5 \mathrm{~nm}$ thick InAs cap layer $\left(N_{D}=5 \times 10^{18} \mathrm{~cm}^{-3}\right)$. The MC parameters for the electron transport simulation in the involved materials can be found in Ref. [9].

In the $\mathrm{MC}$ simulations, impact ionization is included for electrons in the $\Gamma$ valley by means of the Keldysh approach [15]: the probability per unit time of having an impact ionization event is given by $P(E)=S\left[\left(E-E_{t h}\right) / E_{t h}\right]^{2}$ if $E>E_{t h}$, and $P(E)=0$ if $E<E_{t h}, E$ being the electron kinetic energy in the $\Gamma$ valley, $E_{t h}$ the ionization threshold energy and $S$ a measure of the softness or hardness of the threshold. When an impact ionization process takes place, a hole in the heavy-hole valence band and an electron in the $\Gamma$ valley emerge, while the original electron remains in the $\Gamma$ valley. All the details for the modelling of hole transport can be found in Ref. [14].

For a proper analysis of kink effect, hole recombination must be also taken into account. Therefore, we use a simple model in which hole recombination is considered to take place with a characteristic time $\tau_{\text {rec }}$ (i.e., with a probability $1 / \tau_{\text {rec }}$ ). We have performed simulations with $\tau_{\text {rec }}=0.05 \mathrm{~ns}$, since with this value of $\tau_{\text {rec }}$ (jointly with those of $E_{\text {th }}$ and $S)$ the experimental static output characteristics are adequately reproduced [14]. Moreover, we have considered other values of $\tau_{\text {rec }}$ in order to check its influence on the noise behavior of the device.

Regarding the noise calculations, the fluctuations of the current flow through the drain electrode are studied by means of the autocorrelation function. The corresponding spectral density of drain-current fluctuations is determined by Fourier transform of the mentioned autocorrelation function [13]. Due to the presence of the isolated gate, the 
gate current and thus the corresponding noise performance are extremely low for a proper analysis with our MC model.

\section{Results and discussion}

Figure 2 shows the simulated (a), (b) extrinsic and (c), (d) intrinsic $I$ - $V$ characteristics of the InAs-HEMT in presence and absence of impact ionization. In figures 2(a) and 2(b) the experimental $I-V$ curves [7] have been included for comparison, and in figures 2(c) and $2(\mathrm{~d})$ the case of $\tau_{\mathrm{rec}}=0.02 \mathrm{~ns}$ has been added to check the influence of the recombination time on the static characteristics. In order to carry out the comparison of the measured results (extrinsic) with those obtained from the simulation (intrinsic), drain and source parasitic resistances, associated with metallizations and part of the ohmic regions not included in the simulation domain, have been incorporated into the original MC results. The best fit has been obtained for $R_{S}=0.13 \Omega \cdot \mathrm{mm}$ and $R_{D}=0.38 \Omega \cdot \mathrm{mm}[9,14]$. A remarkable agreement between experimental and simulated results is reached when considering impact ionization in the simulations with values for the involved parameters of $E_{t h}=0.41 \mathrm{eV}, S=10^{12} \mathrm{~s}^{-1}$ and $\tau_{\text {rec }}=0.05 \mathrm{~ns}$, as mentioned previously. A notable increase of $I_{D}$ takes place starting from a value of $V_{D S}$ high enough for the onset of impact ionization [about $V_{D S}=0.3 \mathrm{~V}$, figure 2(b)], leading to a threshold voltage shift at high $V_{D S}$ [figure 2(a)]. The relative $I_{D}$ increase with respect to the case of absence of impact ionization is smaller for a shorter recombination time [figures 2(c) and 2(d)].

MC simulations provide an insight into the microscopic processes taking place inside the devices, in terms of which the kink effect has been explained. Figure 3 shows the potential profile along the channel near the spacer (a), near the buffer (b), and the sheet electron density with and without considering impact ionization in the simulation (c), for $\tau_{\text {rec }}=0.05 \mathrm{~ns}, V_{D S}=0.4 \mathrm{~V}$ and $V_{G S}=-0.6 \mathrm{~V}$. Holes generated by impact ionization in the drain region of the channel (where the electric field, and consequently the electron energy, is higher) accumulate mostly at the drain side of the buffer, which leads to the difference in the channel potential profile with respect to the case of absence on impact ionization, as observed in figures 3(a) and 3(b). This occurs because most of the generated holes descend the energy step present in the valence band at the heterojunction between the InAs channel and the AlSb buffer. Then, the energy of the 
holes decreases due to scattering mechanisms, so that the attracting force of the gate potential and the negative surface charge at the recess is not sufficient for them to surmount back the energy barrier in the valence band and thus return to the channel. This pile up of positive charge lowers the potential barrier that controls the current through the channel, mainly near the buffer side [figures 3(a) and 3(b)], so that the channel is further opened, with the consequent increase in the electron density [figure 3(c)] and drain current. The rise of $I_{D}$ is basically due to this enhancement in the electron flow through the channel, since the number of electrons/holes generated by impact ionization is very low so as to provide a significant contribution to $I_{D}$.

The kink effect is a source of excess noise. Generation of holes by impact ionization and their further recombination lead to fluctuations in the pile up of positive charge located in the buffer of the device. These charge fluctuations are strongly coupled to the drain current by the induced fluctuations on the potential barrier that controls the transport of electrons through the channel. Thus, an important increase of the drain-current noise is expected to take place concurrently with the kink in the $I_{D^{-}} V_{D S}$ curves. The cutoff of this excess noise should thus be related to the relaxation time of the hole accumulation fluctuations.

Figure 4 shows the spectral density of drain current fluctuations $S_{I D}(f)$ in presence and absence of impact ionization, for (a) $V_{D S}=0.4 \mathrm{~V}, \tau_{\text {rec }}=0.05 \mathrm{~ns}$, and different $V_{G S}$, and (b) $V_{G S}=-0.4 \mathrm{~V}, V_{D S}=0.4 \mathrm{~V}$, and different $\tau_{\text {rec. }}$ Both impact ionization and hole recombination lead to a positive long-time contribution in the drain-current correlation function, which originates a significant increase of the low-frequency plateau in $S_{I D}(f)$, not present when impact ionization is removed from the simulations. The characteristic cutoff frequency of the plateau in $S_{I D}(f)$ is linked to the impact ionization rate and the hole recombination probability, this being $1 / \tau_{\text {rec. }}$. Moreover, the longer is $\tau_{\text {rec}}$, the higher is the noise level. At frequencies beyond the cutoff, the noise remains practically independent of $\tau_{\text {rec}}$, exhibiting the typical peak related to plasma oscillations in the highly doped regions of the structure. For the case in which $\tau_{\text {rec }}=0.05 \mathrm{~ns}$ the drain-current autocorrelation-function has a very long-time tail, which leads to spurious mathematical contributions to the $S_{I D}(f)$ curve, which must be not taken into account for the global explanation [13]. Note that in the case of absence of 
impact ionization the low-frequency plateau shows no cutoff before the noise increase related to plasma oscillations.

The low-frequency value of the spectral-density of drain-current fluctuations $S_{I D}(0)$ is presented in figure 5 as a function of (a) $V_{G S}$ for $V_{D S}=0.4 \mathrm{~V}$, and (b) $V_{D S}$ for $V_{G S}=-0.6 \mathrm{~V}$, and different values of $\tau_{\text {rec }}$. The case of absence of impact ionization is also included for comparison. $S_{I D}(0)$ is found to increase with $V_{D S}$ due to the higher current and number of impact ionization events taking place at larger gate-drain potential difference. The increase of $S_{I D}(0)$ is especially pronounced for high $V_{G S}$. This is the opposite behavior to that found for InGaAs/InAlAs HEMTs [10], where, when the channel is opened, the maximum electron energy, and thus the number of impact ionization events, is reduced due to the lower gate-to-drain potential (although the electron density in the channel is larger). In the case of InAs/AlSb HEMTs, the band gap of the channel material and also the threshold energy $E_{t h}$ are much smaller than for InGaAs/InAlAs devices, so that the impact ionization probability remains significant for higher values of $V_{G S}$. Furthermore, impact ionization events take place not only near the maximum of electron energy (under the gate electrode) but all along the drain side of the channel, where the electron velocity is higher when increasing $V_{G S}$. This leads to higher values of $I_{D}$ and thus $S_{I D}(0)$ when increasing $V_{G S}$. Moreover, the values of $S_{I D}(0)$ are larger for higher $\tau_{\text {rec }}$ due to the longer time that holes remain inside the device before recombination, which leads to a higher hole density and a longer characteristic time of hole density relaxation. As observed in figure $5, S_{I D}(0)$ depends on $V_{D S}$ and $V_{G S}$ in a similar way to that shown by the kink-related increase of $I_{D}$ (more pronounced for higher $V_{D S}$ and $V_{G S}$ ). However, the relative increase of $S_{I D}(0)$ with respect to its value when impact ionization is not considered in the simulations is much higher than that of $I_{D}$. Thus, although the kink effect in the $I_{D}-V_{D S}$ curves is hardly detectable for values of $V_{D S}$ between $0.2 \mathrm{~V}$ and $0.3 \mathrm{~V}$ [figure 2(b)], $S_{I D}(0)$ already exhibits a significant increase with respect to its value in absence of impact ionization. For example, the relative increase of $I_{D}$ when impact ionization takes place with respect to case of absence of impact ionization is about $12 \%$ for $V_{G S}=-0.6 \mathrm{~V}, V_{D S}=0.3 \mathrm{~V}$ and $\tau_{\text {rec }}=0.05 \mathrm{~ns}$, while the increase of $S_{I D}(0)$ is around $83 \%$. This indicates that when HEMTs are biased in the vicinity of the kink 
onset, even if the static behavior of the transistors is not strongly perturbed, their noise performance can be extremely degraded, since drain noise is very sensitive to the dynamics of holes generated by impact ionization. This fact further confirms that the increase of $S_{I D}$ associated with the kink effect is mainly due to the appearance of an excess noise connected with the strong drain current fluctuations initiated by the variations of the hole concentration in the buffer.

\section{Conclusions}

We have presented an MC-based study of the degradation introduced by the kink effect in the noise performance of an isolated-gate InAs/AlSb HEMT. With this purpose, we have analyzed the drain current spectral density calculated by the Fourier transform of the drain current fluctuations correlation function. The fluctuations of the hole pile-up originated by impact ionization are linked to those of the drain current through the fluctuations of the potential barrier that controls the electron transport through the channel. As a consequence, the spectral density of the drain-current presents a lowfrequency plateau whose cutoff frequency is linked to the impact ionization rate and the hole recombination probability. The drain noise is very sensitive to the dynamics of holes generated by impact ionization; indeed, the noise performance of InAs/AlSb HEMTs can be seriously degraded when they are biased in the vicinity of the kink onset, even if the static behavior of the transistors is not perturbed.

\section{Acknowledgments}

This work has been partially supported by the Dirección General de Investigación (MICINN) through Project TEC2010-15413, by the Consejería de Educación, Junta de Castilla y León through Project GR270. 


\section{References}

[1] Boos J B, Kruppa W, Bennett B R, Park D, Kirchoefer S W, Bass R and Dietrich, H B 1998 AlSb/InAs HEMT's for Low-Voltage, High-Speed Applications IEEE Trans. Electron Devices 451869

[2] Bolognesi C R, Dvorak M W, and Chow D H 1999 Impact ionization suppression by quantum confinement: effects on the $\mathrm{DC}$ and microwave performance of narrow band-gap channel InAs/AlSb HEMT's IEEE Trans. Electron Devices 46 826

[3] Kruppa W, Boos J B, Bennett B R, Papanicolaou N A, Park D and Bass R 2007 Low-Frequency Noise in A1Sb/InAs and related HEMTs IEEE Trans. Electron Devices 541193

[4] Ma B Y, Bergman J, Chen P, Hacker J B, Sullivan G, Nagy G and Brar B 2006 InAs/AlSb HEMT and Its Application to Ultra-Low-Power Wideband High-Gain Low-Noise Amplifiers IEEE Trans. Microwave Theory Tech. 544448

[5] Kim D-H and del Alamo J A 2010 30-nm InAs PHEMTs with $\mathrm{f}_{\mathrm{t}}=644 \mathrm{GHz}$ and $\mathrm{f}_{\max }=681 \mathrm{GHz}$ IEEE Electron Device Lett. 31806

[6] Malmkvist M, Lefebvre E, Borg M, Desplanque L, Wallart X, Dambrine G, Bollaert S, and Grahn J 2008 Electrical characterization and small-signal modeling of InAs/AlSb HEMTs for low-noise and high-frequency applications IEEE Trans. Microwave Theory Tech. 562685

[7] Lefebvre E, Malmkvist M, Borg M, Desplanque L, Wallar X, Dambrine G, Bollaert S, and Grahn J 2009 Gate-recess technology for InAs/AlSb HEMTs IEEE Trans. Electron Devices 561904

[8] Mateos J, González T, Pardo D, Hoel V, Happy H, Cappy A 2000 Monte Carlo simulator for the design optimization of low-noise HEMTs IEEE Trans. Electron Devices 471950

[9] Rodilla H, González T, Pardo D, and Mateos J 2009 High-mobility heterostructures based on InAs and InSb: a Monte Carlo study J. Appl. Phys. 105 113705(1-6)

[10] Rodilla H, González T, Moschetty G, Grahn J and Mateos J 2011 Dynamic Monte Carlo study of isolated-gate InAs/AlSb HEMTs Semicond. Sci. Technol. 26 025004 
[11] Rodilla H, González T, Moschetty G, Grahn J and Mateos J 2012 Monte Carlo study of the noise performance of isolated-gate InAs/AlSb HEMTs Semicond. Sci. Technol. 27015008

[12] Vasallo B G, Mateos J, Pardo D and González T 2003 Monte Carlo study of the kink effect in short-channel InAlAs/InGaAs high electron mobility transistors $J$. Appl. Phys. 944096

[13] Vasallo B G, Mateos J, Pardo D and González T 2004 Kink-effect related noise in InAlAs/InGaAs high electron mobility transistors J. Appl. Phys. 958271

[14] Vasallo B G, Rodilla H, González T, Moschetty G, Grahn J and Mateos J 2010 Monte Carlo study of kink effect in isolated-gate InAs/AlSb high electron mobility transistors J. Appl. Phys. 108 094505(1-5)

[15] V. Fischetti 1991 Monte Carlo simulation of transport in technologically significant semiconductors of the diamond and zinc-blende structures - part I: homogeneous transport IEEE Trans. Electron Devices 38634 


\section{Figure captions}

Figure 1. Schematic topology of the HEMT under analysis.

Figure 2. $\mathrm{MC}$ (a), (b) extrinsic and (c), (d) intrinsic $I-V$ characteristics for the InAs/AlSb HEMT in presence and absence of impact ionization, being $E_{t h}=0.41 \mathrm{eV}, S=10^{12} \mathrm{~s}^{-1}$ and different $\tau_{\text {rec. }}$. Experimental results of the similar fabricated structure are plotted in (a), (b) for comparison. In the $I_{D}-V_{G S}$ curves, $V_{D S}$ is (a) $0.6 \mathrm{~V}$ and (c) $0.4 \mathrm{~V}$ for the top curves and the potential step is $\Delta V_{D S}=-0.1 \mathrm{~V}$ in both cases. In the $I_{D}-V_{D S}$ curves, $V_{G S}$ is (b) $-0.45 \mathrm{~V}$ and (d) $-0.40 \mathrm{~V}$ for the top curves and the potential step is (b) $\Delta V_{G S}=-0.1 \mathrm{~V}$ and (d) $\Delta V_{G S}=-0.2 \mathrm{~V}$.

Figure 3. MC profiles along the channel of (a) potential near the spacer and (b) potential near the buffer, and (c) sheet electron density with and without considering impact ionization in the simulation, being $V_{D S}=0.4 \mathrm{~V}, V_{G S}=-0.6 \mathrm{~V}$. $E_{\text {th }}=0.41 \mathrm{eV}, S=10^{12} \mathrm{~s}^{-1}$ and $\tau_{\text {rec }}=0.05 \mathrm{~ns}$.

Figure 4. $\mathrm{MC}$ values of $S_{I D}(f)$ in presence and absence of impact ionization, for (a) $V_{D S}=0.4 \mathrm{~V}, \tau_{r e c}=0.05 \mathrm{~ns}$, and different $V_{G S}$, and (b) $V_{G S}=-0.4 \mathrm{~V}, V_{D S}=0.4 \mathrm{~V}$, and different $\tau_{\text {rec. }} E_{\text {th }}=0.41 \mathrm{eV}, S=10^{12} \mathrm{~s}^{-1}$.

Figure 5. MC values of $S_{I D}(0)$ in presence and absence of impact ionization, as a function of (a) $V_{G S}$ being $V_{D S}=0.4 \mathrm{~V}$, and (b) $V_{D S}$ being $V_{G S}=-0.6 \mathrm{~V}$. $E_{t h}=0.41 \mathrm{eV}$, $S=10^{12} \mathrm{~s}^{-1}$ and different $\tau_{\text {rec }}$. 


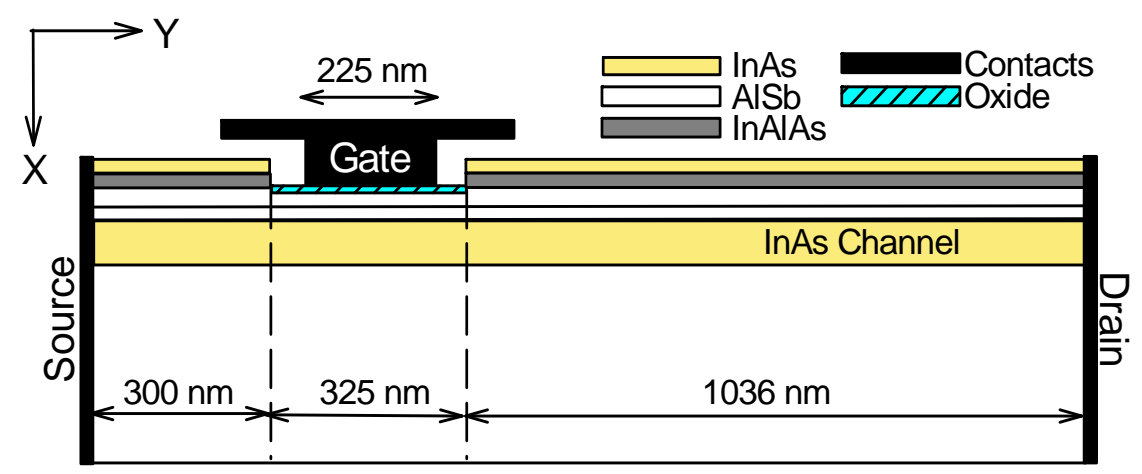

Figure 1 
Extrinsic $l-V$ characteristics

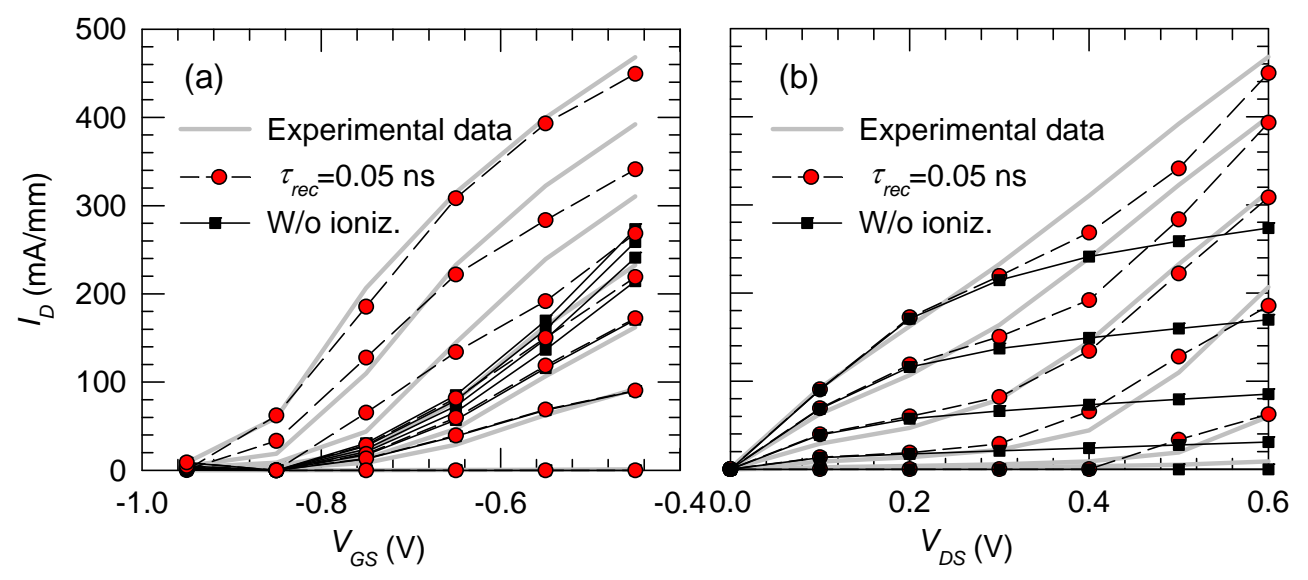

Intrinsic $I-V$ characteristics

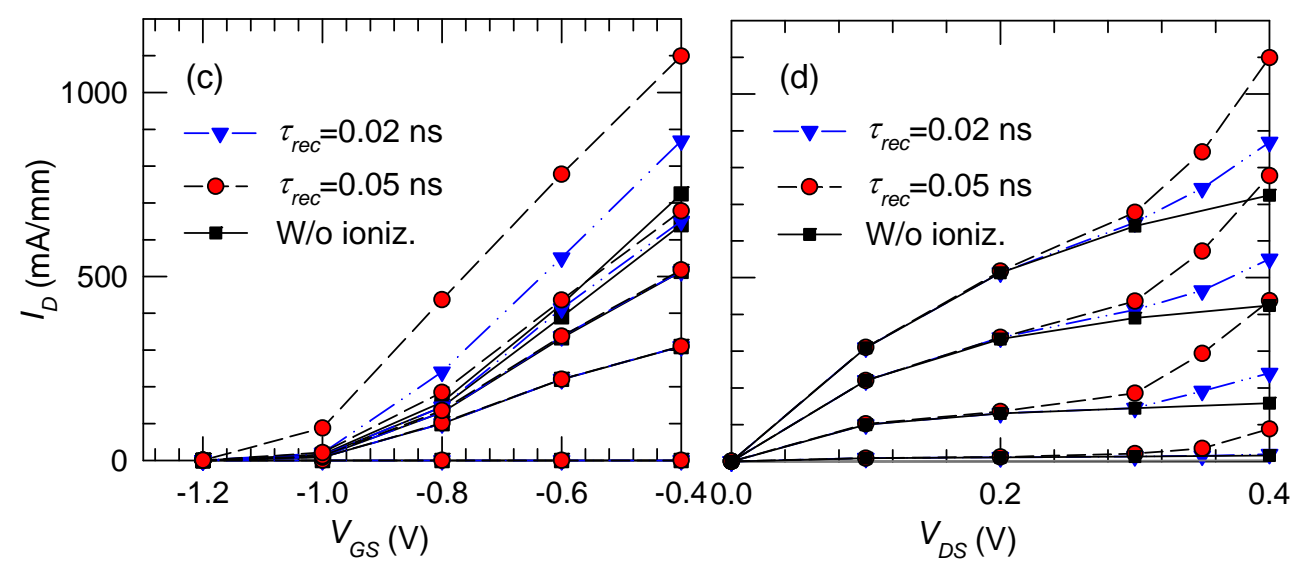

Figure 2 


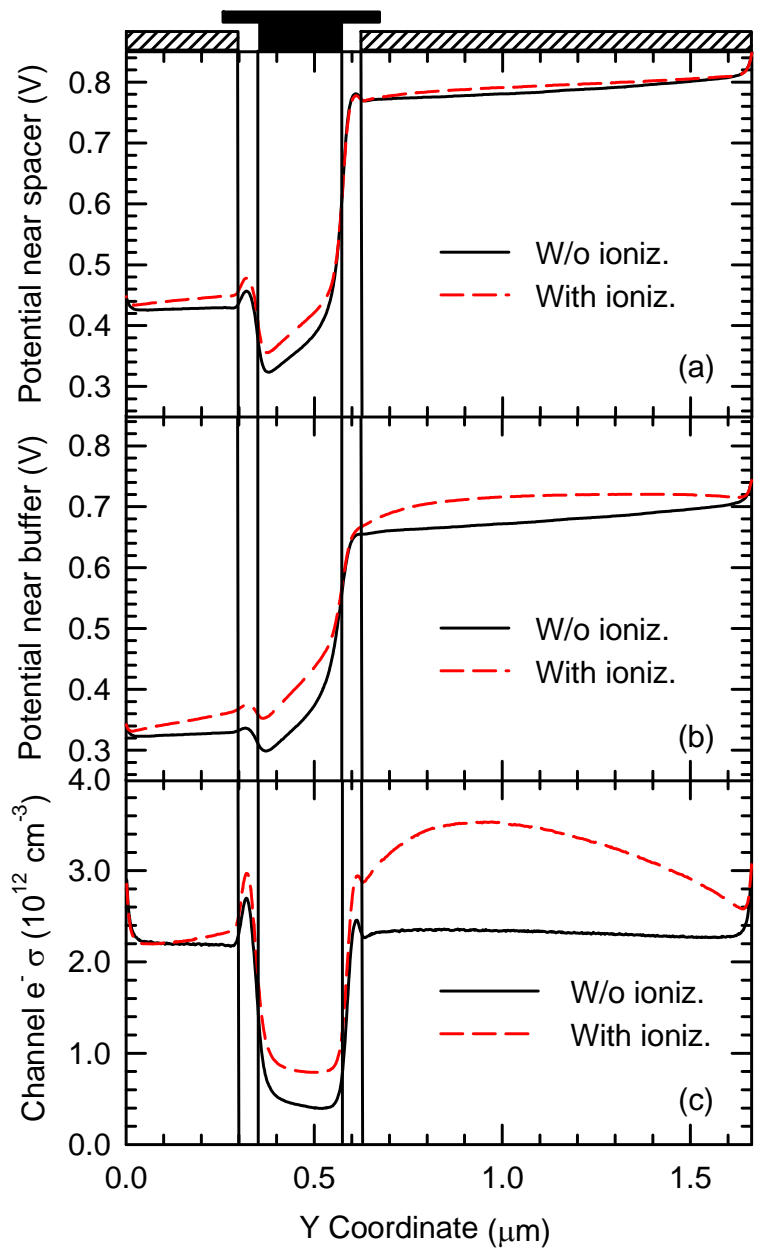

Figure 3 


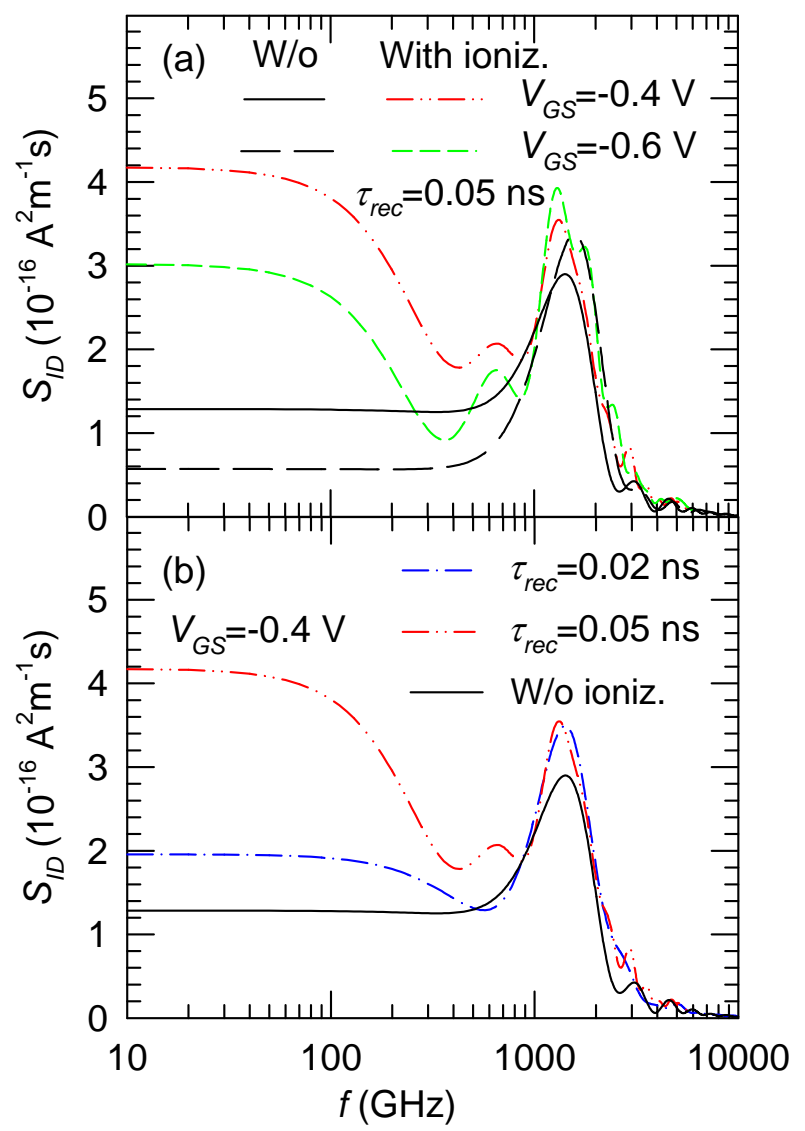

Figure 4 

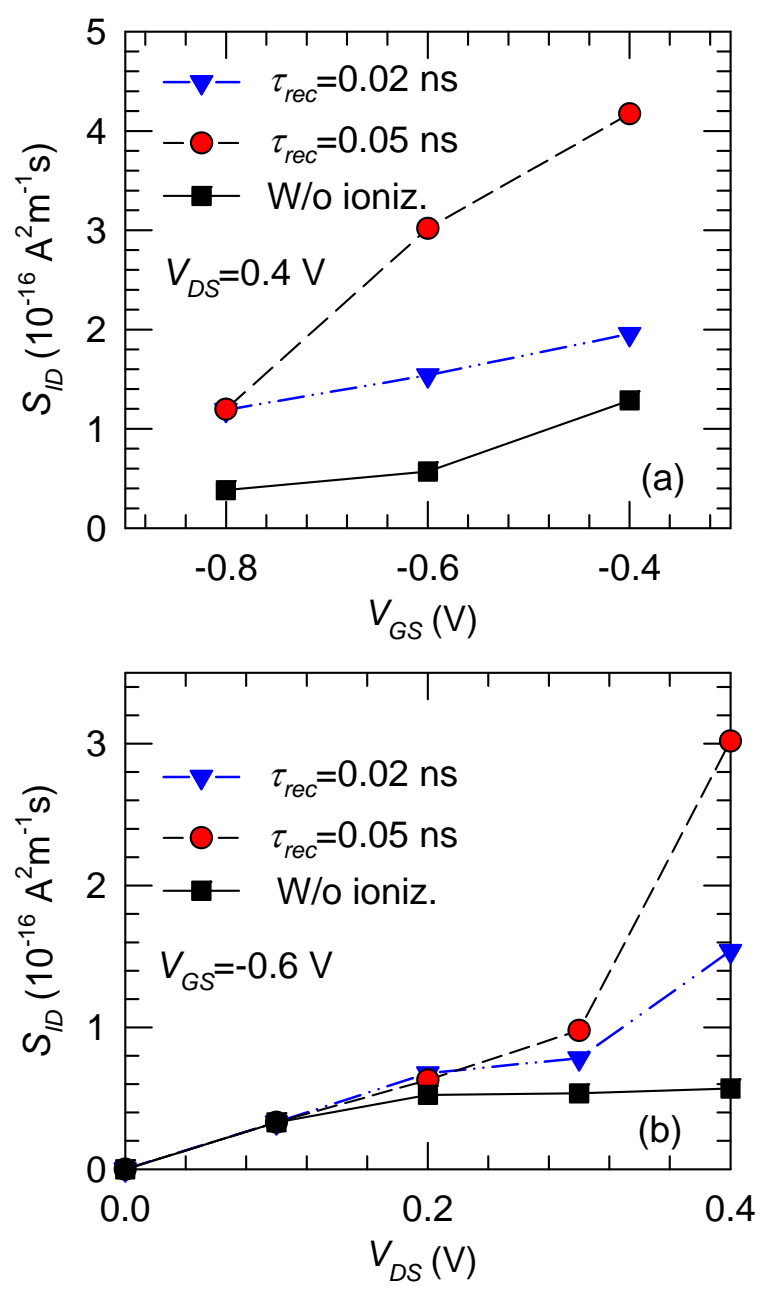

Figure 5 